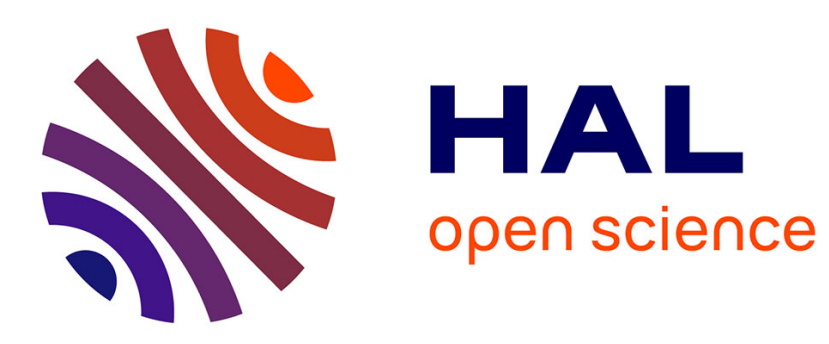

\title{
Influence of search neutrality on the economics of advertisement-financed content
}

Pierre Coucheney, Giuseppe d’Acquisto, Patrick Maillé, Maurizio Naldi, Bruno Tuffin

\section{- To cite this version:}

Pierre Coucheney, Giuseppe d'Acquisto, Patrick Maillé, Maurizio Naldi, Bruno Tuffin. Influence of search neutrality on the economics of advertisement-financed content. ACM Transactions on Internet Technology, 2014, pp.20. hal-00908569

\section{HAL Id: hal-00908569 \\ https://hal.inria.fr/hal-00908569}

Submitted on 25 Nov 2013

HAL is a multi-disciplinary open access archive for the deposit and dissemination of scientific research documents, whether they are published or not. The documents may come from teaching and research institutions in France or abroad, or from public or private research centers.
L'archive ouverte pluridisciplinaire HAL, est destinée au dépôt et à la diffusion de documents scientifiques de niveau recherche, publiés ou non, émanant des établissements d'enseignement et de recherche français ou étrangers, des laboratoires publics ou privés. 


\section{Influence of search neutrality on the economics of advertisement-financed content}

PIERRE COUCHENEY, Université de Versailles / PRiSM

GIUSEPPE D'ACQUISTO, Garante per la protezione dei dati personali

PATRICK MAILLÉ, Institut Mines-Telecom / Telecom Bretagne

MAURIZIO NALDI, Università di Roma Tor Vergata

BRUNO TUFFIN, Inria

The recently raised search neutrality debate questions the ranking methods implemented by search engines: when a search is performed, do they (or should they) display the web pages ordered according to the quality-of-experience (relevance) of the content? In this paper, we analyze that question in a setting when content is offered for free, content providers making revenue through advertising. For content providers, determining the amount of advertising to add to their content is a crucial strategic decision. Modeling the trade-off between the revenue per visit and the attractiveness, we investigate the interactions among competing content providers as a non-cooperative game, and consider the equilibrium situations to compare the different ranking policies. Our results indicate that when the search engine is not involved with any high-quality content provider, then it is in its best interest to implement a neutral ranking, which also maximizes user perceived quality-of-experience and favors innovation. On the other hand, if the search engine controls some high-quality content, then favoring it in its ranking and adding more advertisement yields a larger revenue. This is not necessarily at the expense of user perceived quality, but drastically reduces the advertising revenues of the other content providers, hence reducing their chances to innovate.

Categories and Subject Descriptors: K.6.0 [Management of computing and information systems]: Economics

General Terms: Management, Performance

Additional Key Words and Phrases: Search neutrality, Economics, Game theory

ACM Reference Format:

Pierre Coucheney, Giuseppe D'Acquisto, Patrick Maillé, Maurizio Naldi, and Bruno Tuffin. ACM V, N, Article A (January YYYY), 20 pages.

DOI : http://dx.doi.org/10.1145/0000000.0000000

\section{INTRODUCTION}

Search engines represent nowadays the dominant way to access content on the web. Their role allows them to exert a remarkable influence on what people see and read on the Internet. Early on, the possibility that, either by accident or by deliberate choice, such influence turns into bias has been noted and whistleblowed against [Introna and Nissenbaum 2000].

Actually, the structure of the market of search engines was such to raise the issue of a strong concentration as early as in 2001, when the Hirschman-Herfindahl concentration

Author's addresses: Pierre Coucheney, Université de Versailles / PRiSM, France, E-mail: pierre.coucheney@uvsq.fr; Giuseppe D'Acquisto, Garante per la protezione dei dati personali, Italy, E-mail: g.dacquisto@garanteprivacy.it; Patrick Maillé, Institut Mines-Telecom / Telecom Bretagne, France, E-mail: patrick.maille@telecom-bretagne.eu; Maurizio Naldi, Università di Roma Tor Vergata, Italy, E-mail: naldi@disp.uniroma2.it; Bruno Tuffin, Inria, France, E-mail: bruno.tuffin@inria.fr.

Permission to make digital or hard copies of part or all of this work for personal or classroom use is granted without fee provided that copies are not made or distributed for profit or commercial advantage and that copies show this notice on the first page or initial screen of a display along with the full citation. Copyrights for components of this work owned by others than ACM must be honored. Abstracting with credit is permitted. To copy otherwise, to republish, to post on servers, to redistribute to lists, or to use any component of this work in other works requires prior specific permission and/or a fee. Permissions may be requested from Publications Dept., ACM, Inc., 2 Penn Plaza, Suite 701, New York, NY 10121-0701 USA, fax +1 (212) 869-0481, or permissions@acm.org.

(c) YYYY ACM 0000-0000/YYYY/01-ARTA $\$ 15.00$

DOI : http://dx.doi.org/10.1145/0000000.0000000 
index (HHI), measuring the degree of competition and defined as the sum of the squares of the market shares - the smaller it is the higher the competition- was 0.116 [Sheu and Carley 2001]. The low barriers for new entrants observed in [Gandal 2001] have turned into the present extremely strong dominance of Google [Telang et al. 2004], with an HHI well in excess of 0.6. The present near-monopoly structure of that market further strengthens concerns about the capability to influence operators located elsewhere in the value chain.

Search engines base their business model on advertising [Levene 2011], providing both organic links and sponsored links. They mark the latters as such and assign them some slots through auctions [Maillé et al. 2012]. Organic links are instead assigned slots through a proprietary ranking mechanism, based on the relevance of the linked webpage for the user's query. However, when ranking organic links, the search engine may include metrics related to its own interest, inducing an unfair result for some links - ranking them below where they should be, and therefore making them rarely reached by users.

Ranking criteria are crucial for the business model of all the stakeholders: search engines, content providers, and advertisers. Content providers wish to improve their ranking among organic links, e.g. through search engine optimization techniques without affecting the quality of the displayed content [Berman and Katona 2013]. Quality is however a main driver for users' choices, entering the competition between organic and sponsored links [White 2013]. The relevance of the webpage's quality for users has been recognized in the case of sponsored links as well [Chen and He 2011; Athey and Ellison 2011], but is in turn influenced by the ranking strategy of the search engine. Search engines may use that influence to their own advantage, distorting both users' choices and the market structure. Acting as intermediaries between buyers and sellers, they can divert their users from their preferred websites to websites for which the search engine has a vested interest [Hagiu and Jullien 2011]. Such a behavior may be labelled as non-neutral, which raises the issue of search neutrality.

A parallel can be made with what currently takes place over the internet, where network providers may unduly discriminate among the service/content providers that use their network (hence the issue of net neutrality [D'Acquisto et al. 2012]). Search neutrality is considered as the next frontier even if net neutrality should prevail [Odlyzko 2009]. In the debate over search neutrality, doubts have been cast about the capability of measures to lead to neutral search results and really protect users against the abuse perpetrated by websites [Grimmelmann 2010]. Critics of the search neutrality approach have even promoted search bias as the product of the competitive process, and the presence of vertical integration (the search engine favouring its own content) as a generally efficient and pro-competitive practice [Manne and Wright 2011]. But the search engine's ranking strategy may adversely affect the market position of content providers, and the debate on search neutrality should consider the impact of a non neutral behavior on the content providers' revenues.

In this paper, we define a model to analyze the influence of search neutrality with regard to the distribution of free content (i.e., content funded by advertising revenues). A typical example is the case of video content. A neutral search engine should rank the results of a search according to the relevance for users of the content, embodied by their quality of experience (QoE), which can be negatively affected by advertising. On the other hand, a non-neutral behavior could allow the search engine (SE) to display results based on the potential revenue it will get from the user consulting them (such a model can encompass the situation where the search engine favors its own content, and the one where it receives payment from CPs to favor their content when a search is performed by a user).

Our objective is to investigate the consequences (in terms of user QoE, content provider and search engine revenue) of the presence or not of a regulation imposing search neutrality. For that purpose, we define a noncooperative game setting among the content providers, which use their level of advertising as a strategic leverage to maximize their revenues. They do so in a framework where the SE (possibly constrained by regulation) decides its ranking policy. We consider both the case of a monopolistic search engine (the rate of requests to 
the SE being assumed independent of the QoE) and that where that rate depends on the quality of the content providers it addresses users to. The ranking policies we examine are based on either quality alone (the neutral approach) or the revenues the content provider transfers to the search engine, possibly weighted through the quality of experience perceived by the user - representing two different non-neutral approaches.

To the best of our knowledge, this paper is one of the very first to try to model mathematically the impact of a non-neutral search engine behavior on the Internet actors, and to analyze it thanks to game theory. The other noticeable reference is [Coucheney et al. 2012], but it is not focusing on the impact of advertisement as we are doing here.

The paper is organized as follows. Section 2 introduces the mathematical models considered in the paper for the quality-of-experience resulting from content with advertising, the influence of ranking on the visit rate of content providers, and the strategies of content providers. In Section 3, we analyze the case where content providers neglect their individual influence on the success of the search engine (the number of requests per time unit), with the advertising strategies of content providers studied as a non-cooperative game. We prove the existence of a Nash equilibrium for that game, whatever the ranking policy. Under a neutral policy, we find that content providers with the same intrinsic quality (the perceived quality in the absence of advertising) are led to reduce their advertising level as the number of competitors grows. Content providers with higher intrinsic quality are instead led to advertise more, getting larger revenues. If the search engine adopts a revenue-based ranking policy, we prove that content providers are led to set their advertising level to the maximum possible value, zeroing the quality perceived by users, regardless of their relationship to the search engine. If the revenue-based policy is mitigated by considering the quality of experience as well, vertically integrated content providers are favored, yielding more advertising and larger revenues. Section 4 treats the case of content providers including their effect on the request arrival rate in their strategic decision, and compares the performance of neutral and non-neutral rankings. In that case, we prove that the Nash equilibrium can be found as the solution of a system of polynomial equations. Under the neutral policy, content providers with the same intrinsic quality set their advertising level so as to halve their quality of experience. Under a non-neutral policy, content providers are instead led to increase their advertising as the number of competitors grows, though their revenues decline until being lower than in the neutral case. The conclusions are given in Section 5.

\section{MODELS FOR THE BEHAVIOR OF THE STAKEHOLDERS}

We consider a single search engine that has to rank different webpages hosting content when a user performs a search. Though we focus on a single search engine, we do account for the possibility that the user selects an alternative search engine, abandoning the strictly monopolistic market structure for search engines. Contents are controlled by different CPs, which can play with the amount of advertisement included in their webpage. Throughout this paper, we consider a given piece of content (e.g., a video sequence, a movie, a TV show) that is searched for by a user and proposed by a set $\mathcal{I}$ of CPs. In this section, we provide models for the behavior of all the stakeholders: users, the search engine, and content providers. A discussion on general challenges, modeling issues and interactions between stakeholders in the field of network economics can be found in [Maillé and Tuffin 2014].

\subsection{User's quality of experience with content providers}

In addition to the sheer quality of the content, the quality of CPs as perceived by users is influenced by several factors: the graphical design of the user interface, the number of clicks needed to reach the content, and the time elapsed before accessing the content (for example, some CPs add an advertising sequence of a few seconds before a video can be watched). In all those cases, advertisements included by a $\mathrm{CP}$ are perceived as a nuisance by users, lowering their overall quality of experience. Advertisements have therefore a two-fold effect: 
they positively affect the content providers' advertising revenues, but negatively affect the QoE perceived by users. We denote by $A_{i}$ the total amount of advertising introduced by $\mathrm{CP} i$, and interpret it in two ways, depending on the considered point of view.

- For the CP: $A_{i}$ is proportional to the advertising revenue that the $\mathrm{CP}$ gets each time a user clicks on the link.

- For users: $A_{i}$ corresponds to a nuisance, and advertisement is supposed to yield a loss of QoE proportional to $A_{i}$.

Through some inessential changes of unit, we consider that CP $i$ earns $A_{i}$ each time its content is accessed, and that the quality of experience $V_{i}$ for the user with $\mathrm{CP} i \in \mathcal{I}$ is

$$
V_{i}=Q_{i}-A_{i},
$$

where $Q_{i}>0$ is the intrinsic quality of the content of $\mathrm{CP} i$ (that experienced by the user if there were no advertisement). We assume that $V_{i}$ is an intrinsic characteristic of the content and advertisement bundle provided by the content provider, depending neither on the decisions taken by the search engine (e.g., its ranking criterion), nor on the user's tastes.

For convenience, we will also use the notation $a_{i}:=A_{i} / Q_{i}$, the relative amount of advertisement introduced by CP $i$. Note also that we limit the advertising level to values that give a non-negative $\mathrm{QoE}$, therefore we have $A_{i} \in\left[0, Q_{i}\right]$, or equivalently $a_{i} \in[0,1]$.

\subsection{User's choice of a search engine}

We focus only on one SE in this paper, but the user may not choose that SE, e.g., if the quality of the results provided by the SE is bad. We summarize that effect through the average request arrival rate $\beta$ (for the considered content) that the $\mathrm{SE}$ receives per time unit. We assume that it depends on the expected QoE of the user with that SE. We use $\beta$ as a proxy for the probability that the user chooses that SE among all the possible choices.

\subsection{SE ranking policies and click-through-rates}

While the user's QoE is determined by both the webpage's intrinsic quality and the amount of advertisement, the ranking criterion has a considerable influence on the behavior of users, and may reduce the visibility of some CPs with good QoE. In general, most users click on one of the links in the highest slots allocated by the SE. We assume in this paper that the link clicked after a search depends only on the ranking determined by the SE based on the scores $s=\left(s_{i}\right)_{i \in \mathcal{I}}$ attributed by the SE to each CP. The SE can adopt one of several score functions, depending on its aim. In this section, we examine the most relevant, subdivided into the two general categories of neutral and non neutral behavior of the search engine.

We do not address separately ranking and the subsequent user clicking behavior here (in terms of click-through rate for each position in the ranking), but rather aggregate those two phenomena by considering that the SE allocates the slots (possibly introducing randomness) based on the set of scores $s$, so that the proportion of clicks on $\mathrm{CP} i$ is

$$
C_{i}=\frac{s_{i}}{\sum_{j \in \mathcal{I}} s_{j}}
$$

Note that the average total number of clicks per search can be different from 1 , since some users may decide not to click any link while other may try several ones. That constant factor can however be considered equal to 1 without loss of generality, since it can be included in the value of $\beta$ (that would then be interpreted as the number of links visited via the SE per time unit). In other words, $C_{i}$ is the probability (conditional on a link being clicked) that $\mathrm{CP} i$ is accessed. That probability describes the overall behavior of the user.

2.3.1. Neutral ranking behavior. Search neutrality should correspond to a situation where $\mathrm{QoE}$ is the only thing that matters when ranking, without any consideration for profit. The 
score considered by the $\mathrm{SE}$ in the neutral case thus equals the QoE, i.e.

$$
s_{i}=V_{i}, \quad \forall i \in \mathcal{I} .
$$

According to (1), the proportion $C_{i}$ of clicks on $\mathrm{CP} i$ is then

$$
C_{i}=\frac{V_{i}}{\sum_{j \in \mathcal{I}} V_{j}}=\frac{Q_{i}\left(1-a_{i}\right)}{\sum_{j \in \mathcal{I}} Q_{j}\left(1-a_{j}\right)} .
$$

2.3.2. Non-neutral ranking strategies. We will say that the SE adopts a non-neutral stance when the scores considered to rank content providers are not the QoE values $\left(V_{i}\right)_{i \in \mathcal{I}}$. In particular, we investigate the case when the ranking criterion adopted by the SE takes into account that the SE obtains a share $b_{i}$ of the advertising revenue of each $\mathrm{CP} i \in \mathcal{I}$. This should then constitute an incentive for the SE to favor CPs with a large $b_{i}$ and large amounts of advertising. More specifically, we consider two possible non-neutral ranking strategies:

- Revenue-based ranking, where the SE ranks content providers on the basis of the revenue it can collect from them rather than the quality experienced by the users. If the search engine receives money by content providers, it has a real interest in favoring those that may generate more revenues, hence to set the ranking scores

$$
s_{i}=b_{i} A_{i}=b_{i} Q_{i} a_{i}, \quad \forall i \in \mathcal{I},
$$

which lead to a click-through rate

$$
C_{i}=\frac{b_{i} Q_{i} a_{i}}{\sum_{j \in \mathcal{I}} b_{j} Q_{j} a_{j}} .
$$

- Weighted-QoE ranking where the SE modifies the neutral ranking rule (based on the user's QoE alone), introducing a bias to favor the CPs for which it has a greater economic interest. The bias is modelled by introducing the corrective factor $b_{i}$ in the neutral rule (2), which can also be interpreted as the share of the CP controlled by the SE: this is directly connected to the search neutrality debate, where search engines can be accused of favoring the contents they (partially) own. The scores considered in that case are then

$$
s_{i}=b_{i} V_{i}=b_{i}\left(Q_{i}-A_{i}\right)=b_{i} Q_{i}\left(1-a_{i}\right), \quad \forall i \in \mathcal{I},
$$

so that the click-through rate is

$$
C_{i}=\frac{b_{i} Q_{i}\left(1-a_{i}\right)}{\sum_{j \in \mathcal{I}} b_{j} Q_{j}\left(1-a_{j}\right)}
$$

\subsection{Content providers: revenues and strategies}

The expected revenues (per time unit) of a content provider $i \in \mathcal{I}$ are denoted by $R_{i}$. Since we are considering free content in this work, CPs' revenues only come from advertising and are proportional to the amount of advertisem ent added to their content, but also to the number of clicks they receive per time unit (that depends on the SE ranking through (1)).

After deducting the fee paid to the $\mathrm{SE}$, the average revenues per time unit of any $\mathrm{CP}$ are

$$
R_{i}=\beta C_{i} A_{i}\left(1-b_{i}\right)=\beta C_{i} Q_{i} a_{i}\left(1-b_{i}\right) .
$$

We remark that the revenue of a $\mathrm{CP} i$ depends on its strategic choice $a_{i}$, but also on the amount of advertising $\left(a_{j}\right)_{j \in \mathcal{I} \neq i}$ set by the other CPs, through the proportion of clicks $C_{i}$ to that $\mathrm{CP}$ defined in (4). Here we model those interactions among CPs as a noncooperative game [Osborne and Rubinstein 1994], where each CP chooses its advertising load $a_{i}$ to maximize its revenues. When studying that noncooperative game, we will distinguish two types of models, where content providers act as either price takers or price setters. 
2.4.1. Price-taking content providers. The search engine market is currently dominated by Google. According to the statistics concerning the period October 2011 through March 2012 the share of Google was $80.39 \%$ (see the data at http://www.statowl.com/search engine_market_share.php), with a normalized Hirschmann-Herfindahl index of 0.66, which is by any means an indicator of strong dominance. Reasoning on a short-term basis, we can expect that this quasi-monopoly situation would remain even if the quality of the results displayed were affected by a change in the ranking policy (from neutral to non-neutral). In our model, this can be interpreted as the rate $\beta$ of requests not being affected by the quality of the results. More precisely, that rate may still vary with the average QoE experienced by users, but CPs do not consider that effect when deciding their advertising policy. That bias can for example stem from a large number of CPs (hence the individual effect of each $\mathrm{CP}$ on $\beta$ is small, and neglected). In that sense, content providers are price takers: they do not consider the effect of their own actions on the global "price" (here, the search rate $\beta$ ).

2.4.2. Price-setting content providers. In this model, content providers do anticipate the effect that their advertising strategies have on the global success of the search engine, embodied by $\beta$. We highlight that dependence by writing $\beta=\beta(a)$, where $a=\left(a_{i}\right)_{i \in \mathcal{I}}$ is the advertising profile of CPs.

As a result, each $\mathrm{CP} i$ will choose its advertising level $a_{i}$ so as to maximize

$$
R_{i}=\beta\left(a_{i}, a_{-i}\right) C_{i}\left(a_{i}, a_{-i}\right) a_{i}\left(1-b_{i}\right),
$$

where $a_{-i}$ is the profile of advertising strategies of all CPs but $i$, i.e., $a=\left(a_{i}, a_{-i}\right)$.

In such a case, CPs are said to be price setters, since they are aware of their contribution to the search rate $\beta$, which may decrease as the amount of advertisement increases. Acting as price setters corresponds to making strategic moves with an eye on the long term.

\section{EQUILIBRIUM ADVERTISING STRATEGIES OF PRICE-TAKING CONTENT PROVIDERS}

In this section, we investigate the case when CPs are price takers, i.e., they treat the total request rate $\beta$ as a constant when determining their advertising strategy. For each ranking policy, we will provide results for the general case, before treating two specific situations:

- the symmetric case, where all CPs are identical,

- the duopoly case where only two CPs compete.

We study the behavior of content providers in response to the ranking strategy of search engines as a non cooperative game. Content providers act as the players using the level of advertising as their strategic leverage to maximize their revenues. The game is solved by searching for a Nash equiibrium, after identifying the best response function of each player.

\subsection{Neutral ranking}

Since the multiplicative factor $\left(1-b_{i}\right)$ is constant, and $\beta$ is considered as constant by CPs in this section, the quantity that the $i$-th content provider intends to maximize, under neutral ranking with the scoring function defined in (2), is the utility proportional to

$$
U_{i}:=\frac{R_{i}}{\beta\left(1-b_{i}\right)}=a_{i} Q_{i} C_{i}=a_{i} Q_{i} \frac{V_{i}}{\sum_{j \in \mathcal{I}} V_{j}}=a_{i} Q_{i} \frac{Q_{i}\left(1-a_{i}\right)}{\sum_{j \in \mathcal{I}} Q_{j}\left(1-a_{j}\right)} .
$$

We can establish here the existence of a (non-trivial) Nash equilibrium for the noncooperative game played among CPs.

Proposition 3.1. When the search engine performs a neutral ranking (i.e., based on relevance), the noncooperative game played by CPs fixing their advertising level has at least one Nash equilibrium $a^{N E} \in(0,1)^{|\mathcal{I}|}$. More precisely, there exists a Nash equilibrium, and 
any Nash equilibrium is such that

$$
1 / 2<1+\phi_{i}-\sqrt{\phi_{i}^{2}+\phi_{i}} \leq a_{i}^{N E}<1,
$$

with $\phi_{i}:=\sum_{j \in \mathcal{I} \backslash\{i\}} \frac{Q_{j}}{Q_{i}}$.

Proof. When all the other CPs set their advertising quantities, the $i$-th content provider seeks the quantity $a_{i}$ maximizing $U_{i}$, that is, its best-response to the others' strategic choices.

The case when all competitors of CP $i$ set their advertising level to the maximum value $a_{j}=1$ is degenerate and is treated first. In that case, $C_{i}=1$ as soon as $a_{i}<1$, and $U_{i}=a_{i} Q_{i}$ is then strictly increasing in $a_{i}$. But $C_{i}$ is not defined for $a_{i}=1$, hence no exact best-response exists. However, that case is not a problem, since the strategy $a_{i}=1$ is dominated for each CP $i$, and strictly dominated when at least one opponent $j$ sets $a_{j}<1$. It is therefore an unlikely situation.

We now consider the case when at least one CP $j \neq i$ sets $a_{j}<1$. In that case, $U_{i}$ is a continuous function of $a_{i}$, as can be easily seen in (8).

We remark that $U_{i}=0$ when $a_{i}=0$ and when $a_{i}=1$, and that $\frac{\partial^{2} U_{i}}{\partial a_{i}^{2}}$ has the same sign as $a_{i} Q_{i}-\left(Q_{i}+\sum_{j \in \mathcal{I} \backslash\{i\}} Q_{j}\left(1-a_{j}\right)\right)$, that is strictly negative. As a result, $U_{i}$ is a strictly concave function of $a_{i}$ on the interval $[0,1]$, and has a unique maximum that is in $(0,1)$.

Therefore, the best response of $\mathrm{CP} i$ is the only solution in $(0,1)$ of the equation

$$
\frac{\partial U_{i}}{\partial a_{i}}=\frac{\partial}{\partial a_{i}}\left[a_{i} Q_{i} \frac{Q_{i}\left(1-a_{i}\right)}{\sum_{j \in \mathcal{I}} Q_{j}\left(1-a_{j}\right)}\right]=0,
$$

which brings a quadratic equation in the advertising quantity

$$
a_{i}^{2}-2\left[1+\sum_{j \in \mathcal{I} \backslash\{i\}} \frac{Q_{j}}{Q_{i}}\left(1-a_{j}\right)\right] a_{i}+1+\sum_{j \in \mathcal{I} \backslash\{i\}} \frac{Q_{j}}{Q_{i}}\left(1-a_{j}\right)=0 .
$$

Of the two solutions of that quadratic equation, the larger has to be discarded, since it would lead to $a_{i}>1$. The other solution is therefore in $(0,1)$, and gives us the best response function for content provider $i$ :

$$
\begin{aligned}
& a_{i}^{\mathrm{BR}}=1+\psi_{i}-\sqrt{\left(1+\psi_{i}\right)^{2}-\left(1+\psi_{i}\right)}=1+\psi_{i}-\sqrt{\psi_{i}\left(1+\psi_{i}\right)}, \\
& \text { with } \quad \psi_{i}:=\sum_{j \in \mathcal{I} \backslash\{i\}} \frac{Q_{j}}{Q_{i}}\left(1-a_{j}\right) .
\end{aligned}
$$

For convenience we define $f(x):=1+x-\sqrt{x(1+x)}$, so that $a_{i}^{\mathrm{BR}}=f\left(\psi_{i}\right)$.

Differentiating $f$, we get for $x>0$

$$
\frac{d f(x)}{d x}=1-\frac{2 x+1}{2 \sqrt{x(1+x)}}=1-\frac{x+1 / 2}{\sqrt{(x+1 / 2)^{2}-1 / 4}}<0,
$$

therefore $f$ is strictly decreasing on $\mathbb{R}^{+}$, and thus

$$
\lim _{x \rightarrow \infty} f(x)=1 / 2<f\left(\phi_{i}\right)<a_{i}^{\mathrm{BR}}=f\left(\psi_{i}\right)<f(0)=1,
$$

with $\phi_{i}:=\sum_{j \in \mathcal{I} \backslash\{i\}} \frac{Q_{j}}{Q_{i}}$.

Now let us consider a small $\epsilon \in(0,1)$, and consider any strategy vector $a$ in the compact set $[0,1-\epsilon]^{|\mathcal{I}|}$. From $(13), \psi_{i} \geq(n-1) \frac{Q_{\min }}{Q_{\max }} \epsilon$, where $Q_{\min }:=\min _{j \in \mathcal{I}} Q_{j}$ and $Q_{\max }:=$ 
$\max _{j \in \mathcal{I}} Q_{j}$. From (12), the best-response of each CP $i$ to that strategy vector equals

$$
a_{i}^{\mathrm{BR}}=f\left(\psi_{i}\right) \leq f\left((n-1) \frac{Q_{\min }}{Q_{\max }} \epsilon\right) .
$$

Remark from (14) that the derivative of $f$ is continuous on $(0,+\infty)$ and tends to $-\infty$ at 0 , therefore we can find $\epsilon$ small enough so that $f\left((n-1) \frac{Q_{\min }}{Q_{\max }} \epsilon\right) \leq f(0)-\epsilon$, using the continuity of $f$. Since $f(0)=1$, we obtain that the best-response correspondence

$$
G:\left\{\begin{aligned}
{[0,1-\epsilon]^{|\mathcal{I}|} } & \mapsto[0,1]^{|\mathcal{I}|} \\
a & \mapsto\left(a_{i}^{\mathrm{BR}}\right)_{i \in \mathcal{I}}
\end{aligned}\right.
$$

is such that $f\left([0,1-\epsilon]^{|\mathcal{I}|}\right) \subset[0,1-\epsilon]^{|\mathcal{I}|}$. Since $G$ is continuous and $[0,1-\epsilon]^{|\mathcal{I}|}$ is a compact convex subset of $\mathbb{R}^{|\mathcal{I}|}$, from Brouwer's fixed point theorem, it has a fixed point that constitutes a Nash equilibrium with strategies $a_{i} \in[0,1)$.

The lower bound on the equilibrium strategies directly comes from the lower bound of the best-reply (see Equation (15)).

Though not amenable to an analytical solution, the collection of best response functions (12) for all the content providers builds a system of nonlinear equations in the $a_{i}$ 's, which can be solved numerically to obtain the Nash equilibria points.

3.1.1. Symmetric case. We can consider the special case where all content providers have the same intrinsic quality (symmetric case). By setting $Q_{i}=Q$ in Equation (11), the utility for the $i$-th provider assumes the simple form

$$
U_{i}=a_{i} \frac{1-a_{i}}{\sum_{j \in \mathcal{I}}\left(1-a_{j}\right)} .
$$

In that case, the game can be entirely solved:

Proposition 3.2. When the search engine performs a neutral ranking, the noncooperative game played by CPs fixing their advertising level has the unique Nash equilibrium

$$
a_{i}^{N E}=\frac{n}{2 n-1} \quad \forall i \in \mathcal{I}, n:=|\mathcal{I}| .
$$

Proof. The revenue optimization procedure leads to the equation

$$
\left(1-2 a_{i}\right) \sum_{j \in \mathcal{I}}\left(1-a_{j}\right)+a_{i}\left(1-a_{i}\right)=0 .
$$

This implies that for any $i, k \in \mathcal{I}$, we have

$$
\left(1-2 a_{i}\right) \sum_{j \in \mathcal{I}}\left(1-a_{j}\right)+a_{i}\left(1-a_{i}\right)=\left(1-2 a_{k}\right) \sum_{j \in \mathcal{I}}\left(1-a_{j}\right)+a_{k}\left(1-a_{k}\right),
$$

which yields $\quad\left(a_{k}-a_{i}\right)\left(a_{k}+a_{i}-1+2 \sum_{j \in \mathcal{I}}\left(1-a_{j}\right)\right)=0$.

But from Proposition 3.1, we know that at a Nash equilibrium $a_{j}>1 / 2$ for all $j \in \mathcal{I}$, hence the right factor is strictly positive, and $a_{i}=a_{k}$ : Nash equilibria are necessarily symmetric, of the form $a_{i}=a$ for all $i \in \mathcal{I}$. Plugging that condition into (17), we obtain a unique equilibrium, where the optimal advertising quantity $a$ for any provider is as in (16).

The Nash equilibrium advertising level of (16) is a decreasing function of the number of content providers: each content provider is led to stuff less advertisements as the competition 
level (number of content providers) grows. In the limit, when the number of competitors becomes very large, we have the optimal advertising quantity that cuts by half the QoE with respect to the upper bound represented by intrinsic quality

$$
a_{\lim }=\lim _{n \rightarrow \infty} a=\frac{1}{2} \text {. }
$$

In the symmetric case, the utility for each content provider is

$$
U_{i}=a \frac{1-a}{n(1-a)}=\frac{1}{2 n-1},
$$

while the cumulated utility of the bunch of content providers (recall that the revenue of each provider is its utility multiplied by $\beta(1-b))$ is

$$
U=\sum_{i \in \mathcal{I}} U_{i}=\frac{n}{2 n-1} .
$$

Since utilities are proportional to revenues (recall that $R_{i}=\beta(1-b) U_{i}$ ) and can be taken as a proxy for them, that last expression shows that the aggregated revenue shrinks as the number of players grows: the overall utility reduces by $1 / 3$ when there are just two providers, but by $1 / 2$ when the number of players gets very large.

3.1.2. Duopoly case. Another special case of interest is duopoly, where just two content providers (with different quality) are present. In fact, the presence of high fixed costs reducing profit margins may raise barriers to the entrance of new players and favour a monopoly or duopoly situation (a case of high fixed costs and low marginal costs is presented in [Naldi and D'Acquisto 2008]). In this duopoly case, the best response functions (12) become

$$
\begin{aligned}
& a_{1}=1-\frac{Q_{2}}{Q_{1}}\left(1-a_{2}\right)\left[\sqrt{1+\frac{Q_{1}}{Q_{2}} \frac{1}{1-a_{2}}}-1\right] \\
& a_{2}=1-\frac{Q_{1}}{Q_{2}}\left(1-a_{1}\right)\left[\sqrt{1+\frac{Q_{2}}{Q_{1}} \frac{1}{1-a_{1}}}-1\right] .
\end{aligned}
$$

Again, we can solve that system of nonlinear equations numerically, finding the Nash equilibrium as the intersection (if any) between the best responses being drawn on the same figure, as in Figure 1 (left). The best-response functions for the duopoly case are shown when the providers have equal intrinsic quality. The same shape is observed in all the other cases we have examined. Though the curves cross in two points, the solution that leads to $a_{1}=a_{2}=1$ is to be discarded, since as explained in the proof of Proposition 3.1 there is no real best-response for $\mathrm{CP} i$ when the opponent $j \neq i$ sets $a_{j}=1$, and moreover the strategy $a_{i}=1$ is dominated, and strictly dominated as soon as $a_{j}<1$.

In Figure 1 (right), we see how the Nash equilibrium point moves as the differences in quality between the two providers change. A content provider with higher intrinsic quality can increase its advertising load. In the symmetric case $\left(Q_{1}=Q_{2}\right)$, we obtain the Nash equilibrium point $a_{1}=a_{2}=2 / 3$ from Equation (16) with $n=2$.

3.1.3. Numerical study. We now go back to the general asymmetric case of $n$ content providers and assess the presence and characteristics of Nash equilibrium in typical scenarios. For that purpose, we consider two types of repartition of the intrinsic quality among CPs: linear and geometric. Without loss of generality, we sort the CPs in decreasing order of quality: CP 1 exhibits the largest intrinsic quality, and $\mathrm{CP} n$ has the lowest one. We can define the quality of the generic $i$-th content provider as a function of the two bounds $Q_{1}$ 


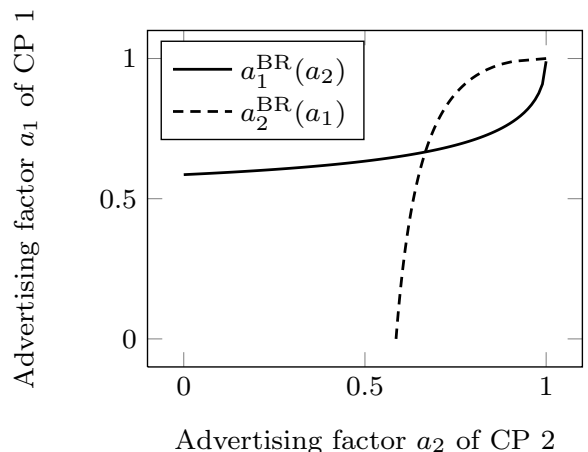

(a) Best response functions

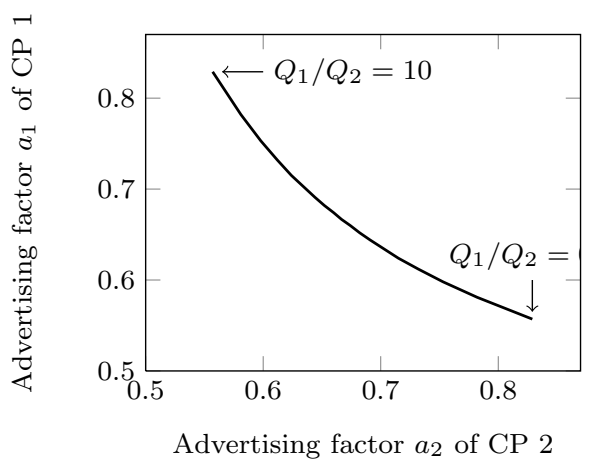

(b) Nash equilibrium points

Fig. 1. Best response functions in the symmetric duopoly case (left) and locus of Nash equilibrium points in a duopoly, both under neutral behavior

and $Q_{n}$. In the linear model, the intrinsic quality of the $i$-th content provider is

$$
Q_{i}=Q_{1}-\frac{i-1}{n-1}\left(Q_{1}-Q_{n}\right) .
$$

In the geometric model, we have instead

$$
Q_{i}=Q_{1} \exp \left(-\frac{i-1}{n-1} \ln \left(Q_{1} / Q_{n}\right)\right) .
$$

Note that we consider here that the share of benefits taken by the search engine is the same for all CPs, i.e. $b_{i}=b$ for all $i \in \mathcal{I}$.

We report here the case of 5 content providers, with the extreme intrinsic quality values $Q_{1}=0.9$ and $Q_{5}=0.1$. We want to see how the intrinsic quality influences the amount of advertisement that is optimal for the content provider (as resulting from the Nash equilibrium). We use the solution (12). Both in the linear and the geometric case, we find a single Nash equilibrium. The relation between the intrinsic quality and the relative amount of advertising (the advertising factor $a_{i}$ ) is shown in Figure 2. Content providers with larger intrinsic quality are led to increase the weight of advertising, though the sensitivity is quite small: a ninefold increase in the intrinsic quality corresponds to an advertising factor increasing by just $17.58 \%$ in the linear model and by $21.94 \%$ in the geometric one.

Utility (and therefore, the revenue of the CP) is also affected by the intrinsic quality, as shown in Figure 2 (right). Though both trends are approximately linear, the more uneven repartition of qualities in the geometric case leads to wider imbalances in the repartition of utilities. While the ratio $Q_{\max } / Q_{\min }$ of extreme intrinsic qualities is 9 in both cases, the range of utility $U_{\max }-U_{\min }$ is larger for the geometric repartition (though the high-to-low ratio for utility is 8.63 for the linear case and just 8.41 for the geometric one)

After examining the impact of the intrinsic quality on the individual strategic decisions about the level of advertising, we now turn our attention to the impact on the quality perceived by users. For the $i$-th content provider, the introduction of advertisement brings its quality down from the intrinsic value $Q_{i}$ to the $\mathrm{QoE} V_{i}=Q_{i}\left(1-a_{i}\right)$. In the above example, we have considered a wide range for intrinsic quality values. That would be the range of quality values experienced by users in the absence of advertisement. We can examine if the introduction of advertisement (which is the leverage through which content providers seek their maximal profits) magnifies those differences in quality or levels them out.

In order to assess that impact, we employ the Herfindahl-Hirschman Index, which measures the concentration of a market among a number of competitors (i.e., the level of com- 

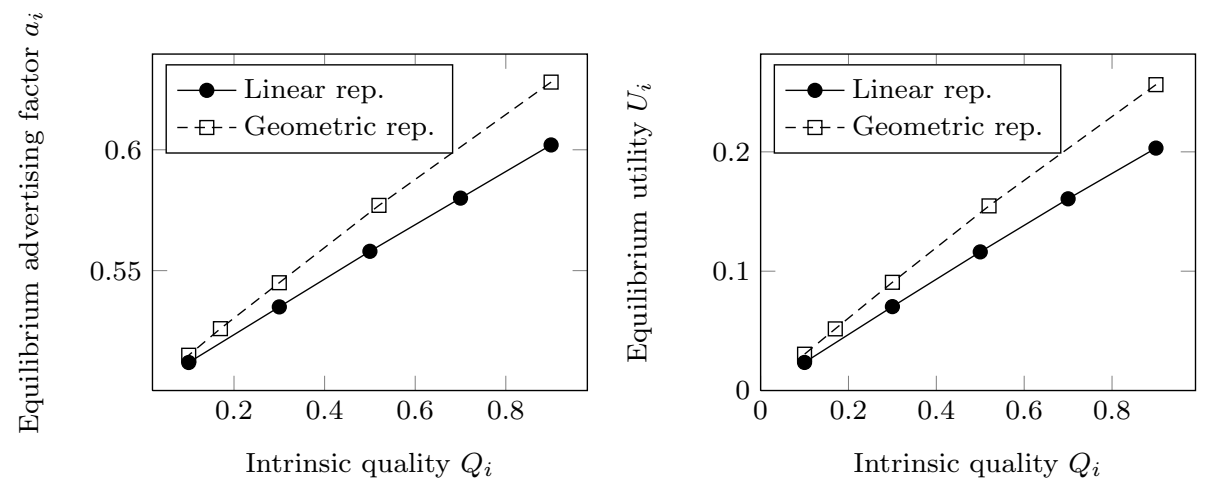

Fig. 2. Impact of the intrinsic quality on the game outcome (left) and utility (right), for price-taking CPs and a neutral ranking

petition) and is the most sensitive among such indicators [Naldi 2003]. Given a set of market shares $\left\{m_{1}, m_{2}, \ldots, m_{n}\right\}$, which satisfy the constraint $\sum_{i \in \mathcal{I}} m_{i}=1$, the HHI is

$$
\mathrm{HHI}=\sum_{i \in \mathcal{I}} m_{i}^{2},
$$

and lies in the interval $[0,1]$. Higher values of the HHI indicate a larger degree of concentration (hence a lower level of competition). Here we do not have the market shares (expressed as fractions of the overall revenues), but we consider utility values as their proxy. In fact, utilities are proportional to revenues, the proportionality constant being equal for all competitors. After normalizing the utility values to their sum, we get figures equal to the market shares. In order to distinguish the HHI computed by using utilities to that computed using the market shares (though they lead to the same numerical result), we use the definition

$$
\mathrm{HHI}_{\mathrm{U}}=\sum_{i \in \mathcal{I}}\left(\frac{U_{i}}{\sum_{j \in \mathcal{I}} U_{j}}\right)^{2}=\frac{\sum_{i \in \mathcal{I}} U_{i}^{2}}{\left(\sum_{i \in \mathcal{I}} U_{i}\right)^{2}} .
$$

In addition to measuring the concentration of the market, we can use the HHI to measure the attraction power through quality values. In fact, quality works as a magnet for users: contents of higher quality attract more users, and differences in quality correspond to different capabilities to attract users. In this context, the HHI can be used to measure the degree of concentration of quality among the content providers. If we replace the market shares in the definition (19) with quality values, we obtain the two quality-based HHI

$$
\begin{aligned}
\mathrm{HHI}_{\mathrm{Q}} & =\sum_{i \in \mathcal{I}}\left(\frac{Q_{i}}{\sum_{j \in \mathcal{I}} Q_{j}}\right)^{2}=\frac{\sum_{i \in \mathcal{I}} Q_{i}^{2}}{\left(\sum_{i \in \mathcal{I}} Q_{i}\right)^{2}}, \\
\mathrm{HHI}_{\mathrm{V}} & =\sum_{i \in \mathcal{I}}\left(\frac{V_{i}}{\sum_{j \in \mathcal{I}} V_{j}}\right)^{2}=\frac{\sum_{i \in \mathcal{I}} V_{i}^{2}}{\left(\sum_{i \in \mathcal{I}} V_{i}\right)^{2}},
\end{aligned}
$$

employing respectively the intrinsic quality and the perceived quality. Larger values of $\mathrm{HHI}_{\mathrm{Q}}$ mean higher imbalances in quality. By comparing $\mathrm{HHI}_{\mathrm{Q}}$ (prior to including advertising) with $\mathrm{HHI}_{\mathrm{V}}$ (including advertisements), we can assess the effect of advertising choices of all content providers on the structure of the quality offer. 
Table I. Attraction power concentration (price-taking CPs)

\begin{tabular}{|c|c|c|}
\hline & Linear repartition & Geometric repartition \\
\hline $\mathrm{HHI}_{\mathrm{Q}}$ & 0.264 & 0.305 \\
\hline $\mathrm{HHI}_{\mathrm{V}}$ & 0.253 & 0.279 \\
\hline $\mathrm{HHI}_{\mathrm{U}}$ & 0.261 & 0.298 \\
\hline
\end{tabular}

We report the results in Table I. Under both the linear and the geometric model, the HHI index is larger (though not by much) for the intrinsic quality than for the QoE: the introduction of advertisement brings along a slight levelling of the quality perceived by the user. In Table I, we also report the HHI (denoted by $\mathrm{HHI}_{\mathrm{U}}$ ) pertaining to market concentration. We see that the concentration is somewhat intermediate between that of the intrinsic quality and that of the perceived quality: the repartition of utility is less affected by the introduction of advertisement than the quality perceived by users.

\subsection{Non-neutral behavior: revenue-based ranking}

When the ranking is only based on the potential revenue for the SE, and scores are taken from (3), with the position $X_{i}:=b_{i} Q_{i}$, the utility of the content provider is given by:

$$
U_{i}=\beta A_{i} C_{i}\left(1-b_{i}\right)=\beta \frac{1-b_{i}}{b_{i}} \frac{a_{i}^{2}\left(b_{i} Q_{i}\right)^{2}}{\sum_{j \in \mathcal{I}} a_{j} Q_{j} b_{j}}=\frac{1-b_{i}}{b_{i}} \frac{a_{i}^{2} X_{i}^{2}}{\sum_{j \in \mathcal{I}} a_{j} X_{j}}
$$

The following proposition shows that the non-neutral behavior incentivizes CPs to increase their advertising level with respect to the neutral case. That incentive is indeed twofold, since a larger $a_{i}$ yields more revenue per click, but also attracts more clicks because of the non-neutral ranking (where $s_{i}$ increases with $a_{i}$ ).

Proposition 3.3. When the search engine performs a revenue-based ranking, the noncooperative game played by price-taking CPs fixing their advertising level has a unique Nash equilibrium where each CP sets its advertising level to the maximum possible value, $a_{i}=1$ for all $i \in \mathcal{I}$.

Proof. We simply see that the revenue $R_{i}$ of a $\mathrm{CP} i \in \mathcal{I}$ is strictly increasing in $a_{i}$ : it is indeed null for $a_{i}=0$, and for $a_{i}>0$ it is strictly positive with

$$
\frac{\partial U_{i}}{\partial a_{i}}=\beta \frac{1-b_{i}}{b_{i}} X_{i}^{2} \frac{2 a_{i} \sum_{j \neq i} a_{j} X_{j}+a_{i}^{2} X_{i}}{\left(\sum_{j \in \mathcal{I}} a_{j} X_{j}\right)^{2}}>0 .
$$

We immediately remark that, in that case, the resulting QoE is $V_{i}=0$ for each CP $i$. The revenue of each CP $i \in \mathcal{I}$ becomes $R_{i}=\beta \frac{1-b_{i}}{b_{i}} \frac{X_{i}^{2}}{\sum_{j \in \mathcal{I}} X_{j}}$.

If all CPs transfer to the search engine the same share of their revenue, i.e. $b_{i}=b$ for all $i$, then we obtain $R_{i}=\beta(1-b) \frac{Q_{i}^{2}}{\sum_{j \in \mathcal{I}} Q_{j}}$. Additionally, if as in Section 2.3.1 we consider the symmetric case, where the $n$ content providers have the same intrinsic quality, i.e. $Q_{i}=Q$, then each CP gets a revenue $R_{i}=\beta(1-b) \frac{Q}{n}$.

Note that when $\beta$ varies (increases) with the average QoE for users, that revenue can be much smaller than initially expected by CPs, that as price takers made their strategic choices considering $\beta$ to be a constant. For example, if as in Section 4 we take $\beta$ proportional to the expected user QoE, then the final outcome is a situation where users prefer not to use the search engine (since $\beta=0$ ), and the CPs make no revenue. 


\subsection{Non-neutral behavior: weighted-QoE ranking}

We investigate here the situation where the ranking scores are taken from (5), i.e., the search engine considers the QoE as in the neutral case, but introduces some weights among them so as to favor the CPs it has most interest in.

The resulting proportion of clicks per search on CP $i$ is then

$$
C_{i}=\frac{Q_{i}\left(1-a_{i}\right) b_{i}}{\sum_{j=1}^{n} Q_{j}\left(1-a_{j}\right) b_{j}} .
$$

Accordingly, the utility of the content provider is given by the difference between what it receives through advertising and what it passes to the search engine:

$$
U_{i}=a_{i} C_{i}\left(1-b_{i}\right)=\frac{Q_{i} a_{i} b_{i}\left(1-a_{i}\right)\left(1-b_{i}\right)}{\sum_{j=1}^{n} Q_{j}\left(1-a_{j}\right) b_{j}} .
$$

Again, each content provider seeks to maximize its revenue by setting the quantity of advertising. The analysis follows that carried out in Section 3.1, where for each CP $i$ the parameter $Q_{i}$ is replaced by $b_{i} Q_{i}$. In particular, the best response function of $\mathrm{CP} i$ is then

$$
a_{i}=1+\sum_{\substack{j=1 \\ j \neq i}}^{n} \frac{Q_{j} b_{j}}{Q_{i} b_{i}}\left(1-a_{j}\right)-\sqrt{\left[1+\sum_{\substack{j=1 \\ j \neq i}}^{n} \frac{Q_{j} b_{j}}{Q_{i} b_{i}}\left(1-a_{j}\right)\right] \sum_{\substack{j=1 \\ j \neq i}}^{n} \frac{Q_{j} b_{j}}{Q_{i} b_{i}}\left(1-a_{j}\right) .}
$$

We then have the counterpart of Proposition 3.1.

PROPOSITION 3.4. When the search engine performs a weighted-QoE ranking with weights $\left(b_{i}\right)_{i \in \mathcal{I}}$, the noncooperative game played by price-taking CPs fixing their advertising level has at least one Nash equilibrium $a^{N E} \in(0,1)^{|\mathcal{I}|}$. More precisely, there exists a Nash equilibrium, and any Nash equilibrium is such that

$$
1 / 2<1+\bar{\phi}_{i}-\sqrt{\bar{\phi}_{i}^{2}+\bar{\phi}_{i}} \leq a_{i}^{N E}<1
$$

with $\bar{\phi}_{i}:=\sum_{j \in \mathcal{I} \backslash\{i\}} \frac{b_{j} Q_{j}}{b_{i} Q_{i}}$.

Remark that the symmetric case, where the $n$ content providers have the same intrinsic quality and transfer to the search engine the same share of their utility (i.e., $Q_{i}=Q, b_{i}=b$ ) gives the exact same case as the one analyzed in Section 3.1.1 for the neutral ranking.

3.3.1. Numerical study. We examine now the asymmetric case, solving the game by numerical means. Again, we use the scenario of Section 3.1.3, with 5 content providers whose intrinsic quality follows either a linear or a geometric trend. We consider the case where one content provider is owned by the search engine (it transfers all its utility to it) and all the others pay the same share $b_{i}=0.1$, with two scenarios: the content provider owned by the search engine is that exhibiting either the highest intrinsic quality (Scenario A) or the lowest one (Scenario B), as reported in Table II. We examine the advertising choices made by the content providers and their impact on utility.

We report the results of the game, namely the resulting advertising factor $a_{i}$ determined by each provider, in Figure 3 for the two scenarios. In both cases we see that the content providers not owned by the search engine use an advertising factor that is slightly increasing with the intrinsic quality. Instead, the content provider owned by the search engine is led to substantially increase its advertising load, especially when its intrinsic quality is large: the boost is much higher in Scenario A (where the vertically integrated content provider has an intrinsic quality of 0.9 ) than in Scenario B (where that intrinsic quality is just 0.1). 
Table II. Intrinsic quality and revenue transfer to the search engine in the non-neutral case study

\begin{tabular}{|cc|cc|}
\hline \multicolumn{2}{|c|}{ Intrinsic quality $Q_{i}$} & \multicolumn{2}{|c|}{ Revenue transfer $b_{i}$} \\
\hline Linear & Geometric & Scenario A & Scenario B \\
\hline 0.1 & 0.1 & 0.1 & 1 \\
0.3 & 0.17 & 0.1 & 0.1 \\
0.5 & 0.3 & 0.1 & 0.1 \\
0.7 & 0.52 & 0.1 & 0.1 \\
0.9 & 0.9 & 1 & 0.1 \\
\hline
\end{tabular}

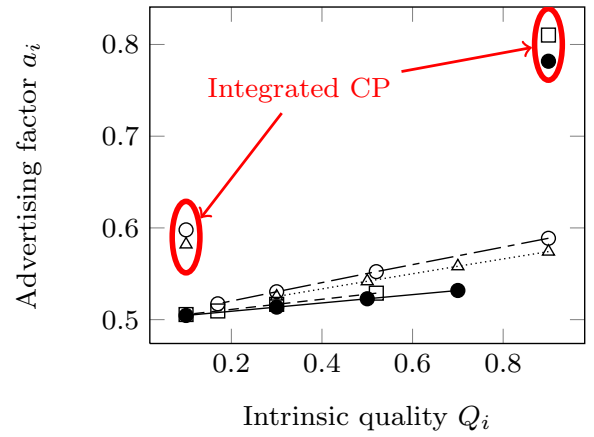

(a) Equilibrium advertising factor

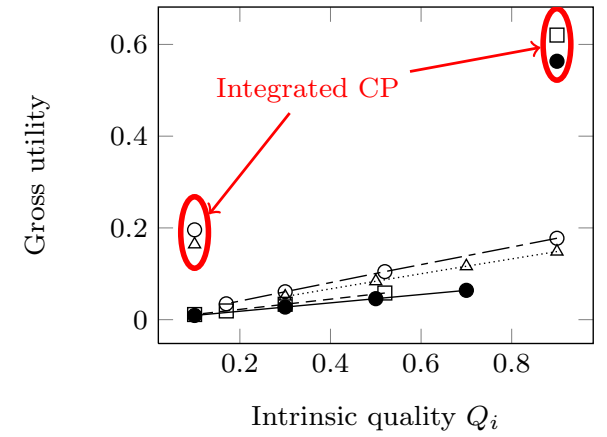

(b) Gross utility

- Sc. A (Linear rep.) - - - Sc. A (Geometric rep.) … $\Delta \cdots$. Sc. B (Linear rep.) ○-Sc. B (Geometric rep.)

Fig. 3. Impact of the intrinsic quality on the advertising factor (left) and gross utility (right), with pricetaking CPs, weighted-QoE ranking.

If we consider the net utility (that remaining after transferring a share to the search engine), the vertically integrated content provider has of course zero utility. But we get a better view of the competition between content providers by considering the gross utility (that obtained prior to paying the tax to the search engine), defined after Equation (20) as

$$
\hat{U}_{i}=\frac{Q_{i} a_{i} b_{i}\left(1-a_{i}\right)}{\sum_{j=1}^{n} Q_{j}\left(1-a_{j}\right) b_{j}} .
$$

In Figure 3 (right), we see that for all providers but that owned by the search engine the utility grows roughly linearly with the intrinsic quality, though at a faster rate in the geometric case. The utility of the content provider owned by the search engine is instead boosted by the larger cash flow it transfers to the search engine, which in turn raises its score in the non-neutral case. The boost is again larger in Scenario A, i.e., when the vertically integrated content provider has the largest intrinsic quality. However, even in Scenario B the boost is enough to bring the vertically integrated content provider to include as much advertisement as the content provider with the highest intrinsic quality.

We can now perform the same concentration analysis as in the neutral case. For the gross utility defined in (21), we can similarly define the HHI

$$
\mathrm{HHI}_{\hat{\mathrm{U}}}=\frac{\sum_{i \in \mathcal{I}} \hat{U}_{i}^{2}}{\left(\sum_{i \in \mathcal{I}} \hat{U}_{i}\right)^{2}} .
$$

The values obtained for HHIs are shown in Table III. By comparing the values of HHI before and after introducing advertisements (i.e., $\mathrm{HHI}_{\mathrm{Q}} \mathrm{AND} \mathrm{HHI}_{\mathrm{V}}$ ), We observe that the introduction of advertisement somewhat levels the quality perceived by users, since the HHI for the QoE is lower than that for the intrinsic quality. Instead, the market concentration 


\begin{tabular}{|c|c|c|c|c|}
\hline Scenarios & A linear & B linear & A geometric & B geometric \\
\hline $\mathrm{HHI}_{\mathrm{Q}}$ & 0.264 & 0.264 & 0.305 & 0.305 \\
\hline $\mathrm{HHI}_{\mathrm{V}}$ & 0.247 & 0.259 & 0.249 & 0.292 \\
\hline $\mathrm{HHI}_{\hat{\mathrm{U}}}$ & 0.644 & 0.227 & 0.708 & 0.261 \\
\hline
\end{tabular}

is significantly altered, in a way that depends on which content provider is owned by the search engine. If the search engine owns the content provider with the highest intrinsic quality (Scenario A), that further boosts its score and its utility, leading to a quite stronger concentration: $\mathrm{HHI}_{\hat{\mathrm{U}}}$ is more than twice as large as $\mathrm{HHI}_{\mathrm{Q}}$. When the search engine owns the content provider with the lowest intrinsic quality (Scenario B), the utility of the latter is likewise pushed up, but this leads to a more balanced repartition and a diminishing HHI.

\section{EQUILIBRIUM ADVERTISING STRATEGIES OF PRICE-SETTING CONTENT PROVIDERS}

Though the present situation of the search engine market is rather close to the monopoly analysed in Section 3, this is neither the general case nor a sure prediction for the future. In this section, we consider that users may select a different search engine. For this general case, we define a game model and find the best response functions. We apply the model to some scenarios and examine the resulting advertising strategies. As in Section 3, we consider separately the case of neutral and non neutral behavior by the search engine.

The propensity of a user to use the search engine under consideration is still represented by $\beta$, assumed here to be proportional to the average QoE of content providers accessed through that search engine (the expected user's QoE when clicking on a link, $C_{i}$ being the probability that the user ends up visiting $\mathrm{CP} i$ ):

$$
\beta=\sum_{i} C_{i} V_{i}
$$

We recall the general expression (7) for $\mathrm{CP} i$ 's revenues

$$
R_{i}=\beta\left(1-b_{i}\right) C_{i} a_{i}
$$

where $C_{i}=s_{i} / \sum_{j \in \mathcal{I}} s_{j}$, and $s_{i}$ is the score credited to $\mathrm{CP} i$ by the SE.

\subsection{Neutral behavior}

As in Section 3.1, the neutral ranking is based on $V_{i}$, hence the proportion of clicks on CP $i$

$$
C_{i}=\frac{V_{i}}{\sum_{j \in \mathcal{I}} V_{j}}
$$

By neglecting the revenue transfer to the search engine in the general expression (22) for the revenues, we can use the utility $U_{i}=\beta C_{i} a_{i}$.

Under that ranking behavior, the revenue of the $i$-th content providers is proportional to

$$
U_{i}=\frac{\sum_{j \in \mathcal{I}} V_{j}^{2}}{\sum_{j \in \mathcal{I}} V_{j}} \frac{V_{i}}{\sum_{j \in \mathcal{I}} V_{j}} a_{i},
$$

which, by setting $X=\sum_{j \in \mathcal{I}} V_{j}$ and $Y=\sum_{j \in \mathcal{I}} V_{j}^{2}$, can be simplified to $U_{i}=V_{i} \frac{Y}{X^{2}} a_{i}$.

The $i$-th content provider optimizes its advertising behavior by maximizing its utility through the appropriate amount of advertising. Remark that $U_{i}=0$ if $a_{i}=0$ or $a_{i}=1$, and $U_{i}>0$ when $a_{i} \in(0,1)$, therefore if the equation $\partial U_{i} / \partial a_{i}=0$ has a unique solution, 
that solution would give the best response function $a_{i}^{*}=f\left(a_{1}, a_{2}, \ldots, a_{i-1}, a_{i+1}, \ldots, a_{n}\right)$. However, since $\partial U_{i} / \partial a_{i}=U_{i} / a_{i}+\partial U_{i} / \partial V_{i} \cdot \partial V_{i} / \partial a_{i}$, and $\partial V_{i} / \partial a_{i}=-Q_{i}$, the optimization equation becomes simply $U_{i} /\left(Q_{i}-V_{i}\right)-\partial U_{i} / \partial V_{i}=0$. By expanding this equation, we obtain the following simplified form of the optimization equation for CP $i$

$$
2 V_{i}\left(V_{i} X-Y\right)\left(1-\frac{V_{i}}{Q_{i}}\right)+X Y\left(1-2 \frac{V_{i}}{Q_{i}}\right)=0 .
$$

If we now replace the full expression for $X$ and $Y$, and rearrange terms, we obtain a fourth-degree polynomial equation in the QoE

$$
\begin{aligned}
& V_{i}^{4}+\left(2 \sum_{j \in \mathcal{I} \backslash\{i\}} V_{j}-\frac{Q_{i}}{2}\right) V_{i}^{3}-\left(\frac{3}{2} Q_{i} \sum_{j \in \mathcal{I} \backslash\{i\}} V_{j}\right) V_{i}^{2}+ \\
& \sum_{j \in \mathcal{I} \backslash\{i\}} V_{j}^{2}\left(\sum_{j \in \mathcal{I} \backslash\{i\}} V_{j}+\frac{Q_{i}}{2}\right) V_{i}-\frac{Q_{i}}{2} \sum_{j \in \mathcal{I} \backslash\{i\}} V_{j} \sum_{j \in \mathcal{I} \backslash\{i\}} V_{j}^{2}=0 .
\end{aligned}
$$

By collecting the $n$ similar expressions for the best response functions of all the content providers, we end up with a system of $n$ polynomial equations, which has to be solved to find Nash equilibria. The system of equations can be solved numerically.

We can consider the special symmetric case by setting $Q_{i}=Q, b_{i}=b$, and $a_{i}=a$ in the general Equation (23), since nothing else depends on the specific content provider:

$$
U=\frac{n V^{2}}{n V} \frac{V}{n V} a=\frac{Q}{n} a(1-a) .
$$

The first order optimality condition is then $\frac{\partial U}{\partial a}=\frac{Q}{n}(1-2 a)=0$, whose solution is $a=\frac{1}{2}$.

\subsection{Non-neutral behavior: revenue-based ranking}

In that case, the utility function of the search engine becomes

$$
U_{i}=C_{i} a_{i} \sum_{j \in \mathcal{I}} C_{j} V_{j}=b_{i} a_{i}^{2} \frac{\sum_{j \in \mathcal{I}} b_{j} Q_{j} a_{j}\left(1-a_{j}\right)}{\left(\sum_{k \in \mathcal{I}} b_{k} a_{k}\right)^{2}}
$$

From the utility maximization condition, we get

$\frac{\partial U_{i}}{\partial a_{i}}=2 b_{i} a_{i} \frac{\sum_{j \in \mathcal{I}} b_{j} Q_{j} a_{j}\left(1-a_{j}\right)}{\left(\sum_{k=1}^{n} b_{k} a_{k}\right)^{2}}+b_{i}^{2} a_{i}^{2} \frac{Q_{i}\left(1-2 a_{i}\right)\left(\sum_{k=1}^{n} b_{k} a_{k}\right)-2 \sum_{j \in \mathcal{I}} b_{j} Q_{j} a_{j}\left(1-a_{j}\right)}{\left(\sum_{k=1}^{n} b_{k} a_{k}\right)^{3}}=0$

which leads to the following optimization equation whose solution should provide the best response function for the $i$-th content provider

$$
2 \sum_{j \in \mathcal{I}} b_{j} Q_{j} a_{j}\left(1-a_{j}\right)\left(1-\frac{b_{i} a_{i}}{\sum_{k=1}^{n} b_{k} a_{k}}\right)+b_{i} a_{i} Q_{i}\left(1-2 a_{i}\right)=0,
$$

giving the following third degree polynomial equation in $a_{i}$ :

$$
-2 b_{i} a_{i}^{3}+\left(b_{i}-4 \sum_{k \neq i} b_{k} a_{k}\right) a_{i}^{2}-3 \sum_{k \neq i} b_{k} a_{k} a_{i}+2 \frac{\sum_{k \neq i} b_{k} a_{k} \sum_{j \neq i} b_{j} Q_{j} a_{j}\left(1-a_{j}\right)}{b_{i} Q_{i}}=0 .
$$

Instead of the numerical approach required in the general case, we can find a simple form of the best response function in the symmetric case. If all the content providers transfer the same percentage of their utility to the search engine and exhibit the same intrinsic quality, 
we expect their best response function to be the same. After setting $b_{i}=b, Q_{i}=Q$, and $a_{i}=a$, and some manipulation, we obtain the symmetric equilibrium advertising level

$$
a=1-\frac{1}{2 n} .
$$

When the number of providers grows, the relative amount of advertising tends towards the saturating value 1 . If we insert the solution (26) in the general expression of utility (25), we get the utility for the symmetric case

$$
U=b a^{2} \frac{n b Q a(1-a)}{n^{2} b^{2} a^{2}}=Q \frac{2 n-1}{4 n^{3}} .
$$

\subsection{Non-neutral behavior: weighted-QoE ranking}

With the scores taken from (5), the utility function of $\mathrm{CP} i$ becomes

$$
U_{i}=b_{i} Q_{i} a_{i}\left(1-a_{i}\right) \frac{\sum_{j \in \mathcal{I}} b_{j} Q_{j}^{2}\left(1-a_{j}\right)^{2}}{\sum_{j \in \mathcal{I}} b_{j} Q_{j}\left(1-a_{j}\right)} .
$$

If we set, for sake of simplicity, $X=\sum_{j \in \mathcal{I}} b_{j} Q_{j}^{2}\left(1-a_{j}\right)^{2}$ and $Y=\sum_{j \in \mathcal{I}} b_{j} Q_{j}\left(1-a_{j}\right)$, the optimization equation can be written as a fourth-degree polynomial equation in $a_{i}$

$$
\left(1-2 a_{i}\right) X Y-2 b_{i} Q_{i}^{2} a_{i}\left(1-a_{i}\right)^{2} Y+b_{i} Q_{i} a_{i}\left(1-a_{i}\right) X=0 .
$$

If we consider the symmetric case, we have $X=n b Q^{2}(1-a)^{2}$ and $Y=n b Q(1-a)$, which, when replaced in (27), give the symmetric equilibrium advertising factor

$$
a=\frac{n}{2 n+1},
$$

which tends to the limit $a=1 / 2$ when the number of providers grows.

In the symmetric case, the utility of each $\mathrm{CP}$ is therefore

$$
U=b Q a(1-a) \frac{n b Q^{2}(1-a)^{2}}{n b Q(1-a)}=b Q^{2} \frac{n(n+1)^{2}}{(2 n+1)^{3}} .
$$

\subsection{Neutral vs Non-neutral Ranking}

We now compare the ranking strategies for the search engine, when the CPs have identical intrinsic qualities. We plot in Figure 4 the equilibrium advertising levels in the symmetric case vs the number of content providers. We refer to the equilibria in (26) and (28) respectively as revenue-based and weighted-QoE scoring. Both functions grow with the number of providers, but achieve different values. When the behavior of the search engine is purely greedy, the advertising factor starts at 0.75 with two CPs and tends to 1 when the number of CPs grows (under tough CP competition the QoE gets very close to zero). Instead, if the scoring function includes the QoE, the optimal advertising factor starts at 0.4 in the duopoly case and, though growing, is upper bounded by the value 0.5: users will get a QoE never lower than what they get in the neutral case (half the intrinsic quality value).

In Figure 4 we also plot the gross utility of each CP. A neutral ranking favors CPs, unless the number of competing CPs becomes large (above 20 when $b=0.1$ ). In that case, the weighted-QoE based ranking would yield a larger utility to CPs.

We now assess the impact of the ranking policy in a non-symmetric setting, with a linear repartition of quality (18), when the number of CPs changes (the geometric distribution yields similar results). We consider two scenarios: the $\mathrm{CP}$ owned by the SE is that with the highest (Figure 5) or the lowest intrinsic quality (Figure 6). We solve the game iteratively. In order to evaluate the impact on users, we define user welfare (or, with a slight abuse of vocabulary, the revenue of users) as being equal to $\beta$, i.e. the propensity of users to use the search engine. We examine the revenue of the $\mathrm{SE}$ (which includes that of its $\mathrm{CP}$ ), the gross 

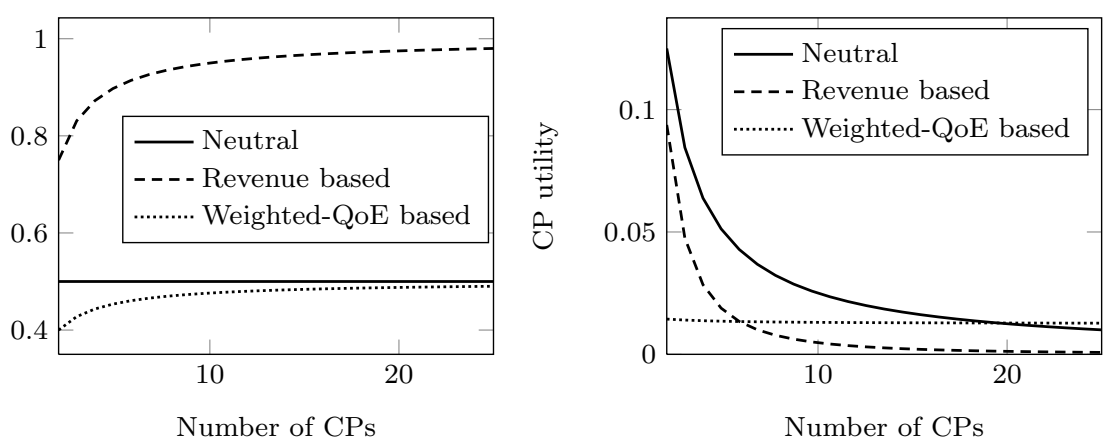

Fig. 4. Nash equilibrium advertising factors (left) and CP utility (right) in the neutral and two non-neutral scenarios, for the symmetric case and price-setting CPs, with $b=0.1$ and $Q=1$

revenue (the aggregated revenue of all CPs but the owned one prior to paying the SE, the revenue of the SE hence including the integrated $\mathrm{CP}$ revenue), and the global revenue, i.e. the sum of both. Notice that, even in the neutral scenario, the equilibrium depends on the $\mathrm{CP}$ vertically integrated with the $\mathrm{SE}$, since their revenue is shared between both entities.

When the smallest CP is integrated with the SE, we observe on Figure 6 that all revenues decrease with the number of CPs. In addition, when there are more than three CPs, the ranking policies are Pareto ordered: the revenue-based ranking is worst for every stakeholder, and the neutral one is always preferred. This suggests that there is no need to enforce search neutrality: even for the search engine it is preferable to implement a neutral ranking.

In the case of the biggest CP integrated, we see on Figure 5 that the comparison is not as clear. The revenues are decreasing w.r.t. the number of players for every stakeholder, except the gross revenue, which increase at first: as the number of CPs grows, they get a bigger and bigger proportion of the global revenue at the expense of the SE. The weightedQoE based ranking provides larger revenues both for the SE and on the overall, probably because owning the biggest quality $\mathrm{CP}$ (rather than the smallest quality one) gives the SE a stronger position in the game so as to adopt a non-neutral ranking. Also, users get a larger welfare for any ranking policy compared to Figure 6 and prefer weighted-QoE based ranking. Since the user's welfare corresponds to the propensity of using the SE, the average relevance is better with the non-neutral ranking. This observation, although not intuitive, shows that the use of non-neutral ranking may result in a Nash equilibrium where less advertisement is set compared to the neutral equilibrium. But this holds here because the $\mathrm{SE}$ owns the most relevant $\mathrm{CP}$, and therefore should not be taken as a valid argument against neutrality. Indeed, one of the objectives of search neutrality is to enable innovation, by making (relevant) new entrants reachable through the SE: in our case a weighted-QoE based ranking goes against that objective, since the average perceived relevance is lower than in the neutral case in Figure 6 (when the best CPs are not owned by the SE).

Finally, note that in both scenarios the revenue of the non-integrated CPs is larger with a neutral ranking, which suggests enforcing neutrality. Indeed, even if a non-neutral stance increases the user's QoE when the SE integrates quality content, it harms the other CPs revenue, possibly preventing them from innovating and improving their quality.

\section{CONCLUSIONS}

This paper provides a mathematical model for the analysis of different ranking policies by search engines, in a context when content providers have to compete for users, and make revenue through advertising. Depending on the ranking adopted, content providers can 

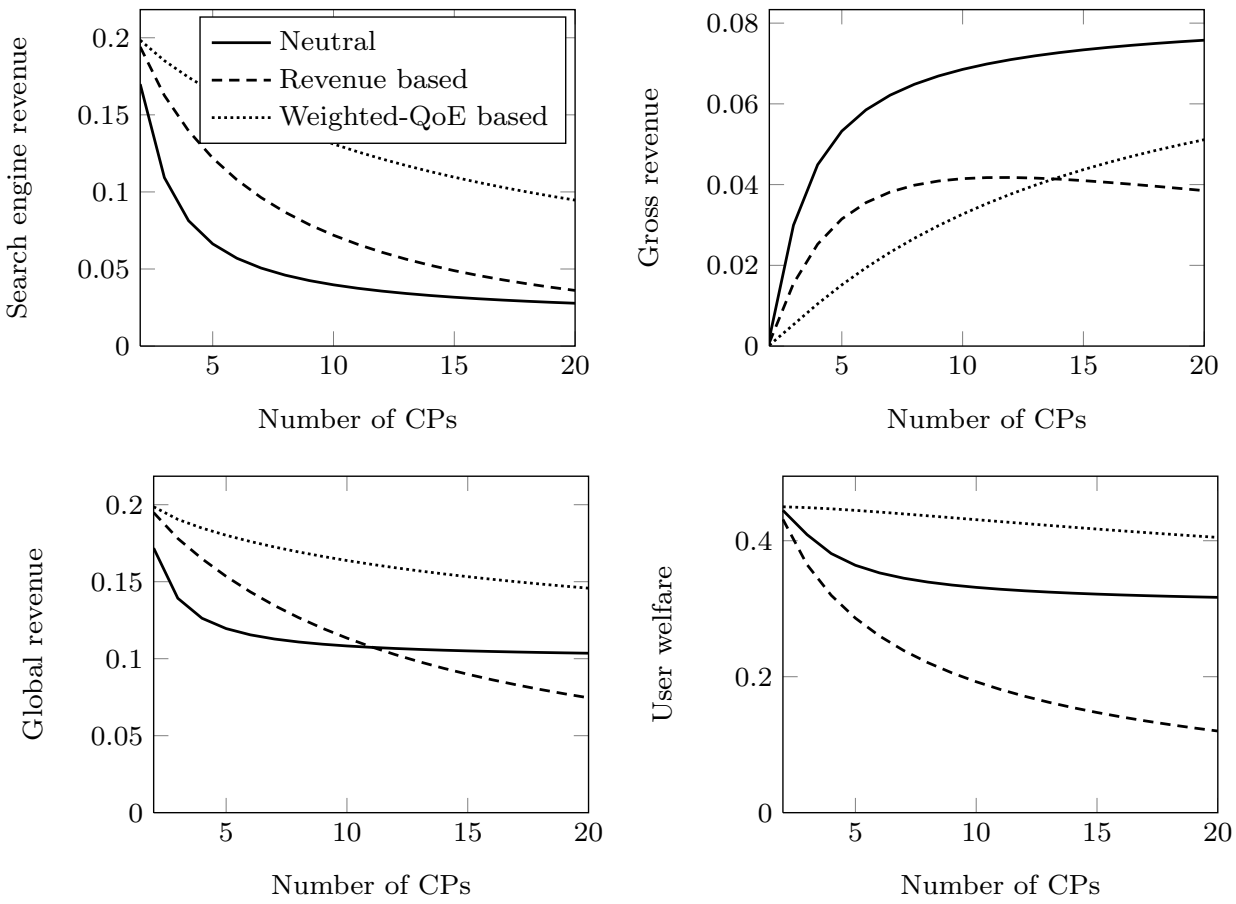

Fig. 5. Utility indices when the highest quality $\mathrm{CP}$ is vertically integrated with the SE.

choose their advertising level, balancing larger advertising revenues against lower qualityof-experience and less users. We have analyzed the noncooperative game played among content providers in different settings, and studied the corresponding equilibria.

Our results indicate that the neutral ranking provides users with the largest perceived quality-of-experience, which is not surprising. But we also observe that such a ranking policy can be preferred as well by a search engine willing to maximize revenue, a less intuitive outcome: this is true if the search engine does not control the best-performing content providers. However, if the search engine integrates quality content, then it can increase its revenue by switching to a non-neutral ranking; this may even benefit to users who will perceive a better quality-of-experience, but would be at the expense of the other content providers, and can then be seen as an impediment to innovation for new entrants.

\section{REFERENCES}

S. Athey and G. Ellison. 2011. Position Auctions with Consumer Search. The Quarterly Journal of Economics 126, 3 (2011), 1213-1270.

R. Berman and Z. Katona. 2013. The Role of Search Engine Optimization in Search Marketing. Marketing Science 32, 4 (2013), 644-651.

Y. Chen and C. He. 2011. Paid Placement: Advertising and Search on the Internet*. The Economic Journal 121, 556 (2011), F309-F328.

P. Coucheney, P. Maillé, and B. Tuffin. 2012. Comparison of search engines non-neutral and neutral behaviors. SIGMETRICS Perform. Eval. Rev. 40, 2 (Oct. 2012), 14-17.

G. D'Acquisto, P. Maillé, M. Naldi, and B. Tuffin. 2012. Impact on retail prices of non-neutral wholesale prices for content providers. In Proceedings of the 8th Euro-NF Conference on Next Generation Internet NGI 2012. IEEE, Karlskrona, Sweden, 111-117.

N. Gandal. 2001. The dynamics of competition in the Internet search engine market. International Journal of Industrial Organization 19, 7 (2001), 1103 - 1117. 

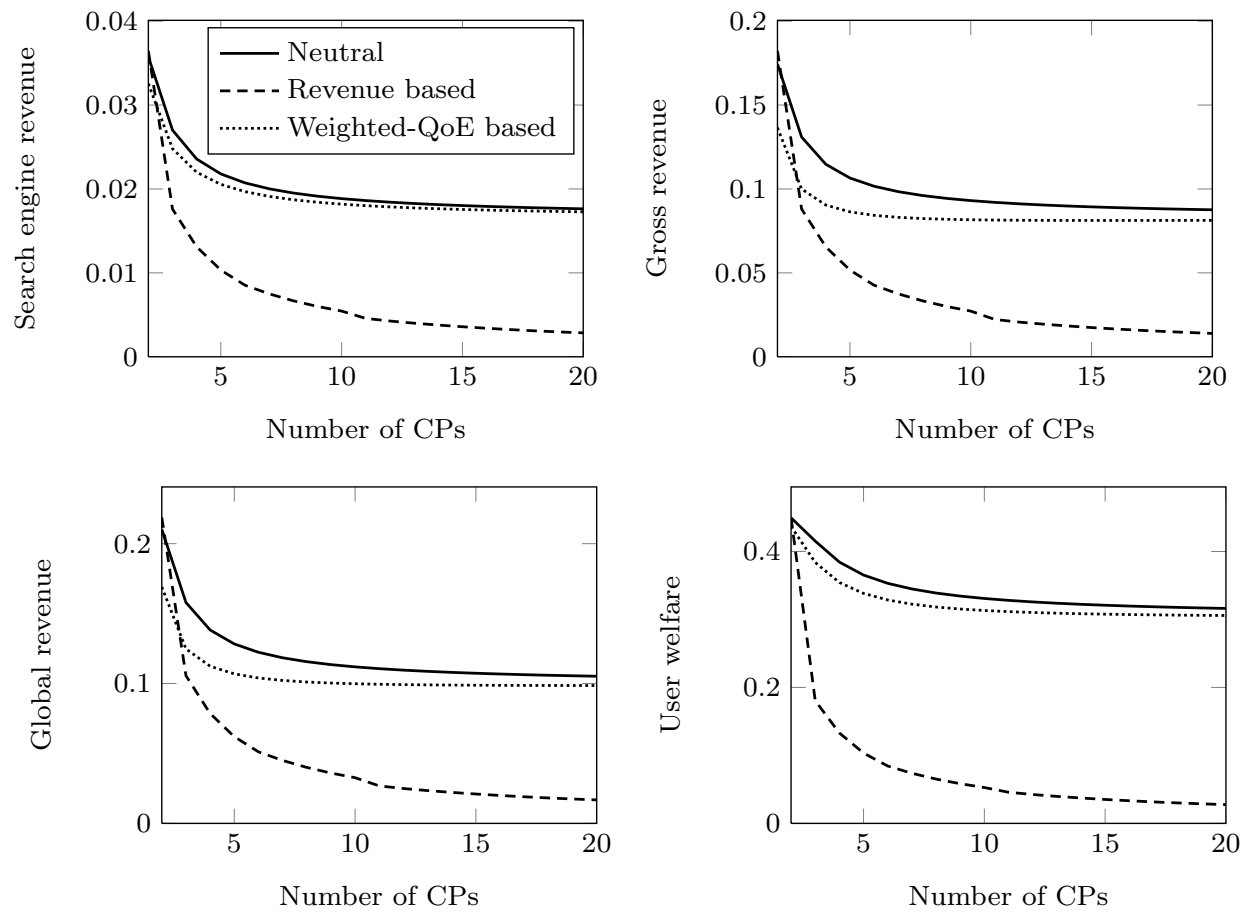

Fig. 6. Utility indices when the smallest quality $\mathrm{CP}$ is vertically integrated with the SE.

J. Grimmelmann. 2010. The next digital decade: Essays on the future of the Internet. TechFreedom, Washington, DC, Chapter Some skepticism about search neutrality, 435-460.

A. Hagiu and B. Jullien. 2011. Why do intermediaries divert search? The RAND Journal of Economics 42, 2 (2011), 337-362.

L. D. Introna and H. Nissenbaum. 2000. Shaping the Web: Why the Politics of Search Engines Matters. Information Society 16, 3 (2000), 169-185.

M. Levene. 2011. An introduction to search engines and web navigation. John Wiley, New Jersey.

P. Maillé, E. Markakis, M. Naldi, G. D. Stamoulis, and B. Tuffin. 2012. Sponsored search auctions: an overview of research with emphasis on game theoretic aspects. Electronic Commerce Research 12 (2012), 265-300. Issue 3.

P. Maillé and B. Tuffin. 2014. Telecommunication Network Economics: From Theory to Practice. Cambridge University Press. In press.

G. A. Manne and J. D. Wright. 2011. If search neutrality is the answer, what's the question? Law and Economics Research Paper Series 11-37. George Mason University.

M. Naldi. 2003. Concentration indices and Zipf's law. Economics letters 78, 3 (2003), 329 - 334.

M. Naldi and G. D'Acquisto. 2008. Performance of the Vickrey auction for digital goods under various bid distributions. Performance Evaluation 65, 1 (2008), 10-31.

A. Odlyzko. 2009. Network Neutrality, Search Neutrality, and the Never-ending Conflict between Efficiency and Fairness in Markets. Review of Network Economics 8, 1 (2009), 40-60.

M. J. Osborne and A. Rubinstein. 1994. A Course in Game Theory. MIT Press, Cambridge, Mass.

T.-R. Sheu and K. Carley. 2001. Monopoly Power on the Web - A Preliminary Investigation of Search Engines. In 29th TPRC Conference. Alexandria, USA.

R. Telang, U. Rajan, and T. Mukhopadhyay. 2004. The Market Structure for Internet Search Engines. J. of Management Information Systems 21, 2 (2004), 137-160.

A. White. 2013. Search engines: Left side quality versus right side profits. International Journal of Industrial Organization (2013), 1-12. 